\title{
Therapeutic benefit of astilbin in the treatment of various forms of livers disorders including hepatitis: Scientific studies of different research work in the medicine
}

\author{
Dinesh Kumar PATEL*
}

Department of Pharmaceutical Sciences, Sam Higginbottom University of Agriculture, Technology and Sciences, Prayagraj, India

Introduction: Flavonoidal compounds are important class phytochemical found to be present in the lots of natural products including numerous herbal plants and their derived secondary metabolites. Astilbin is a pure phytochemical derived from natural plant source and have been well known in the medicine for the treatment of numerous health complications.

Methods: In order to know the health beneficial potential of astilbin in the medicine for the treatment of hepatitis have been evaluated in the present investigation through literature data analysis of different scientific research works. In the literature work effect of astilbin on concanavalin A-induced hepatitis have been investigated and the scientific data have been collected from the research work and analyzed in the present investigation to know the biological importance of astilbin for the treatment of hepatitis. Pharmacological activities of astilbin have been correlated with their medicinal uses for the development of better and effective medicine against hepatitis.

Results: Literature data analysis of different research of astilbin in the scientific filed revealed the biological importance of astilbin in the medicine for the treatment of various liver disorders including hepatitis. Literature study also revealed the medicinal importance of astilbin in the medicine as it significantly inhibited the transaminase activity and TNF-alpha level in the tested organism. Other literature work revealed their significant biological potential in the medicine against hepatitis as it showed improvement of the $\mathrm{T}$ cell-mediated liver injury in the tested organism.

Conclusions: Literature data revealed significant role of astilbin in the treatment of various forms of livers disorders including hepatitis. 